\title{
Vacuum Energy and Renormalization on the Edge
}

\author{
M. Asorey ${ }^{1}$, D. García-Álvarez ${ }^{2}$, J. M. Muñoz-Castañeda ${ }^{1}$ \\ ${ }^{1}$ Departamento de Física Teórica. Facultad de Ciencias. Universidad de Zaragoza, \\ 50009 Zaragoza. Spain \\ 2 Department of Physics, Lancaster University, Lancaster LA1 4YB, United Kingdom \\ E-mail: asorey@unizar.es
}

\begin{abstract}
The vacuum dependence on boundary conditions in quantum field theories is analysed from a very general viewpoint. From this perspective the renormalization prescriptions not only imply the renormalization of the couplings of the theory in the bulk but also the appearance of a flow in the space of boundary conditions. For regular boundaries this flow has a large variety of fixed points and no cyclic orbit. The family of fixed points includes Neumann and Dirichlet boundary conditions. In one-dimensional field theories pseudoperiodic and quasiperiodic boundary conditions are also RG fixed points. Under these conditions massless bosonic free field theories are conformally invariant. Among all fixed points only Neumann boundary conditions are infrared stable fixed points. All other conformal invariant boundary conditions become unstable under some relevant perturbations. In finite volumes we analyse the dependence of the vacuum energy along the trajectories of the renormalization group flow providing an interesting framework for dark energy evolution. On the contrary, the renormalization group flow on the boundary does not affect the leading behaviour of the entanglement entropy of the vacuum in one-dimensional conformally invariant bosonic theories.
\end{abstract}

PACS numbers: 11.10.Hi,11.25.Hf

Keywords: Renormalization group, boundary conditions, vacuum energy. 


\section{Introduction}

The emergence of the dark energy as one of the basic ingredients of the current standard cosmological scenario, and the absence of an even vague understanding of its possible origin, opens a window to the analysis of all possible mechanisms that generate background energy (see e.g. [1] for a review of recent proposals). The main problem is that the apparent value of the dark energy is very tiny compared with any physical energy scale. A second problem is that in a generic quantum field theory there is generation of vacuum energy and any renormalization prescription requires a fine tuning, which is not very convincing without the quantisation of the gravitational interaction.

The guess that dark energy might change with the evolution of the Universe can be understood even if dark energy is just vacuum energy. The finite corrections due to finite size of the causal Hubble domain decrease as the Universe continues to expand.

The aim of this paper is to analyse the variation of these finite size corrections under of renormalization group on the space of boundary conditions for scalar field theories in flat space, although the results are generalisable for more general backgrounds.

The dependence of the vacuum energy on the boundary conditions [2] is well known since the discovery of Casimir effect [3] (see [4,5] and [6] and references therein for recent revisions). However, boundaries might also be considered as a source of new, although peculiar, interactions and therefore can undergo renormalization [7, 8]. The renormalization of boundary conditions modifies the critical behaviour of the theory $[9,10,11]$. In systems with boundaries or defects, the boundary $R G$ flow induces a dynamical behaviour on the boundaries. The dynamics of D-branes in string theory emerges in this way [12].

The renormalization group flow is analysed from a global viewpoint in the most general framework for boundary conditions of scalar field theories introduced in Ref. [13]. In particular, we consider the possible existence of topological transitions [14] induced by the renormalization of boundary conditions or cyclic orbits in the boundary RG flow [15]. The dependence of the finite size corrections to the vacuum energy and vacuum entanglement entropy $[16,17]$ under the boundary $R G$ flow is analysed from a very general perspective.

\section{Boundary conditions in Field Theory}

The action which governs the dynamics of scalar field theory in a bounded domain $\Omega$ of flat space consists of two different of terms, $S(\phi)=S_{B}(\phi)+S_{b}(\phi)$. The first one

$$
S_{B}(\phi)=\frac{1}{2} \int d t \int_{\Omega} \sqrt{g} d^{D} x\left[|\dot{\phi}|^{2}-|\nabla \phi|^{2}-V\left(|\phi|^{2}\right)\right]
$$

is defined in terms of the values of the fields in the bulk. The second term

$$
S_{b}(\varphi)=\frac{1}{2} \int d t \int_{\partial \Omega} \sqrt{g_{\partial \Omega}} d^{D-1} x\left[|\dot{\varphi}|^{2}+\frac{1}{2} \varphi^{*} \partial_{n} \varphi+\frac{1}{2}\left(\partial_{n} \varphi^{*}\right) \varphi-|\nabla \varphi|^{2}\right]
$$


depends only on the values of the fields at the boundary $\partial \Omega^{\dagger} \cdot g_{\partial \Omega}$ denotes the metric induced on the boundary by the bulk flat metric, and $\partial_{n}$ is the normal derivative at the boundary

$$
\varphi=\left.\phi\right|_{\partial \Omega} \quad \partial_{n} \varphi=\left.\partial_{n} \phi\right|_{\partial \Omega} .
$$

The presence of the boundary term $S_{b}$ allows the generation of local classical equations of motion without requiring any specific type of boundary conditions [19, 20]. Indeed, the gradient term

$$
\mathcal{V}=\frac{1}{2} \int_{\Omega}|\nabla \phi|^{2}
$$

can be rewritten as

$$
\mathcal{V}=\frac{1}{2} \int_{\Omega} \phi^{\dagger} \Delta \phi+\frac{1}{2} \int_{\partial \Omega} \phi^{\dagger} \partial_{n} \phi
$$

where $\Delta$ is the Laplace-Beltrami operator $\Delta=-\partial^{\mu} \partial_{\mu}$. In the quantum theory the Laplace-Beltrami operator must have a real spectrum in order to have a selfadjoint Hamiltonian

$$
\mathcal{H}=\frac{1}{2} \sqrt{\Delta+m^{2}}
$$

for the free field theory (The inclusion of interactions does not changes the picture [21]). This means that the classical fields must satisfy boundary conditions which make the operator $\Delta$ selfadjoint. The complete set of boundary conditions which satisfy this requirement [13] are in one-to-one correspondence with the group of unitary operators of the boundary Hilbert space $L^{2}(\partial \Omega, \mathbb{C})$. For any unitary operator $U \in L^{2}(\partial \Omega, \mathbb{C})$, the fields satisfying the boundary condition

$$
\varphi-i \partial_{n} \varphi=U\left(\varphi+i \partial_{n} \varphi\right)
$$

define a domain where $\Delta$ is a selfadjoint operator.

In the case of open strings, the corresponding conformal $1+1$ dimensional scalar field theories is defined on the space interval $\Omega=[0,1] \subset \mathbb{R}$ and there is a large variety of admissible boundary conditions described by the unitary group $\mathcal{M}=U(2)$. The unitary matrices

$$
U_{D}=\left(\begin{array}{cc}
-1 & 0 \\
0 & -1
\end{array}\right) \quad U_{N}=\left(\begin{array}{ll}
1 & 0 \\
0 & 1
\end{array}\right) \quad U_{P}=\left(\begin{array}{ll}
0 & 1 \\
1 & 0
\end{array}\right)
$$

define Dirichlet, Neumann and periodic boundary conditions, which in string theory correspond to a string attached to a D-brane background, free open and closed string theories, respectively.

For higher N-dimensional target spaces, or N-component strings, the set of boundary conditions becomes $\mathcal{M}=U(2 N)$ which includes matrices which interpolate between one single closed string or $\mathrm{N}$ disconnected strings [13]. The topology change is described in this picture by a simple change of boundary conditions in $L^{2}\left(\partial \Omega, \mathbb{C}^{N}\right)$ [14].

${ }^{\dagger}$ We will assume that the boundary is regular and smooth. See e.g. [18] for the peculiarities associated to the presence of irregular boundaries 
If the spectrum of eigenvalues of the unitary operator $U$ does not include the value \pm 1 (i.e. $\pm 1 \notin \mathrm{Sp} U$ ) the boundary condition (7) can be rewritten as

$$
\partial_{n} \varphi=-i \frac{\mathbb{I} \pm U}{\mathbb{I} \mp U} \varphi
$$

which means that only the boundary values of the fields at the boundary can have an arbitrary value $\varphi$ whereas its normal derivative is determined by $U$ and $\varphi$.

The corresponding operator mappings from unitary into selfadjoint operators

$$
A_{ \pm}=-i \frac{\mathbb{I} \pm U}{\mathbb{I} \mp U}
$$

are the celebrated Cayley transforms. The inverse Cayley transform

$$
U=\frac{\mathbb{I} \mp i A_{ \pm}}{\mathbb{I} \pm i A_{ \pm}}
$$

recovers the unitary operator $U$ from their selfadjoint Cayley transforms $A_{ \pm}$.

The condition of $\Delta$ being selfadjoint is necessary but not sufficient to guarantee the unitarity of the corresponding quantum field theory. Indeed, in the case of free field theory the Hamiltonian (6) must be selfadjoint. This requires that the spectrum of $\Delta+m^{2}$ must be not only real but also positive which restricts the set of admissible boundary conditions to a subset $\mathcal{M}$ of $L^{2}(\partial \Omega, \mathbb{C})$.

Because of the existence of the boundary term in (5) the Hamiltonian $\mathcal{H}(6)$ is not selfadjoint if the spectrum of the unitary operator $U$ intersects the following domain of phase factors

$$
S_{m}^{1}=\left\{\mathrm{e}^{2 \alpha i} ;-\pi<\alpha \leq \pi, 0<\alpha<\frac{\pi}{2}-\arctan m^{2}, \text { or } \frac{\pi}{2}<-\alpha<\pi-\arctan m^{2}\right\} .
$$

In any other case, $-m^{2}$ is a lower bound for the spectrum of the operator $\Delta$ and $\mathcal{H}$ is selfadjoint. One possible source of unitarity loss is the existence of edge estates with large negative eigenvalues of operator $\Delta$.

The consistency of the quantum field theory imposes, thus, a very stringent condition on the type of acceptable boundary conditions, even in the case of massive theories in order to prevent this type of pathological behaviour of vacuum energy.

For real scalar fields there is a further condition. $U$ has to satisfy a CP symmetry preserving condition

$$
U^{\dagger}=U^{*}, \quad U=U^{T} .
$$

The usual Neumann and Dirichlet boundary conditions $U= \pm \mathbb{I}$ satisfy this condition. In general, for

$$
U=\left(\begin{array}{ll}
A_{1} & B \\
B^{T} & A_{2}
\end{array}\right)
$$

the condition requires that

$$
\begin{aligned}
& A_{1}=A_{1}^{T}, A_{2}=A_{2}^{T}, A_{1} B^{*}+B A_{2}^{\dagger}=0 \\
& B B^{\dagger}+A_{1} A_{1}^{\dagger}=\mathbb{I}, A_{2} A_{2}^{\dagger}+B^{T} B^{*}=\mathbb{I}
\end{aligned}
$$


In particular, the quasi-periodic condition $\varphi(L)=M^{-1} \varphi(0), \partial_{n} \varphi(L)=M \partial_{n} \varphi(0)$ is also compatible if $M=M^{t}=M^{*}$.

In the case of one single real massless scalar the set of compatible boundary conditions has two connected components: $\mathcal{M}_{0}$ given by the operators of the form

$$
U_{\beta}=\cos \beta \mathbb{I}+i \sin \beta \quad \sigma_{y},
$$

and $\mathcal{M}_{1}$ given by

$$
U_{\alpha}=\cos \alpha \sigma_{z}+\sin \alpha \sigma_{x}
$$

$\mathcal{M}_{0}$ includes Neumann $(\beta=0)$ and Dirichlet $(\beta=\pi)$ conditions; and $\mathcal{M}_{1}$ contains the quasi-periodic boundary conditions

$$
\varphi(L)=\tan \frac{\alpha}{2} \varphi(0) ; \quad \partial_{n} \varphi(L)=\left(\tan \frac{\alpha}{2}\right)^{-1} \partial_{n} \varphi(0)
$$

which include periodic $\left(\alpha=\frac{\pi}{2}\right)$ and antiperiodic $\left(\alpha=-\frac{\pi}{2}\right)$ boundary conditions.

\section{Boundary Conditions and Renormalization Group}

Since boundary conditions appear more naturally in the Schrödinger picture of field theory and the theory is plagued of ultraviolet singularities some doubts were raised about their relevance for the quantum field theory. The pioneer work of Symanzik [21] confirmed the consistence of the standard picture even in presence of bulk renormalizable interactions (see [22] for an explanation of a recent controversy [23]).

Moreover, there is a renormalization of the very boundary conditions because the boundary terms are the source of new interactions.

The renormalization group can be defined in the continuum approach by

$$
\phi_{\Lambda}\left(\frac{x}{\Lambda}\right)=\Lambda^{[\phi]}\left[\phi(x)-\xi_{\Lambda}(x)\right]
$$

by means of a fluctuating field $\xi_{\Lambda}$ with short range fluctuations of order $\frac{1}{\Lambda}$. This implies that the boundary condition

$$
\partial_{n} \varphi=\mathrm{A} \varphi
$$

is renormalised to

$$
\partial_{n} \varphi_{\Lambda}=\mathrm{A}_{\Lambda} \varphi_{\Lambda}
$$

since

$$
\partial_{n} \phi_{\Lambda}\left(\frac{x_{b}}{\Lambda}\right)=\Lambda^{[\phi]+1}\left[\partial_{n} \phi\left(x_{b}\right)-\partial_{n} \xi_{\Lambda}\left(x_{b}\right)\right]=\mathrm{A} \Lambda^{[\phi]+1} \phi\left(x_{b}\right)=\mathrm{A}_{\Lambda} \phi_{\Lambda}\left(\frac{x_{b}}{\Lambda}\right)
$$

with $A_{\Lambda}=\Lambda A$. For more general boundary conditions the continuum renormalization group is given by

$$
\Lambda U_{\Lambda}^{\dagger} \partial_{\Lambda} U_{\Lambda}=\frac{1}{2}\left(U_{\Lambda}^{\dagger}-U_{\Lambda}\right)
$$

or

$$
U_{t}^{\dagger} \partial_{t} U_{t}=\frac{1}{2}\left(U_{t}^{\dagger}-U_{t}\right)
$$


for $\Lambda=\Lambda_{0} \mathrm{e}^{t}$. Fixed points correspond, therefore, to self-adjoint boundary conditions $U^{\dagger}=U$. In particular, Dirichlet and Neumann $(U=\mp \mathbb{I})$ are renormalization group fixed points.

For mixed boundary conditions the RG flows from Dirichlet (UV) toward Neumann (IR) conditions.

$$
U=\mathrm{e}^{2 \mathrm{i} \arctan \mathrm{e}^{-\mathrm{t}}} \mathbb{I} .
$$

Critical exponents can be identified with the eigenvalues of the matrix $U_{c}$ at the fixed points. Since $U_{c}$ is also hermitian all critical exponents are either 1 or -1 and there is no room for cyclic orbits. It is well known, however, that some quantum systems with singular boundaries and singular interactions [15, 24] exhibit cyclic renormalization group flows. Moreover, some topological field theories (e.g. Russian doll models) present a similar behaviour [25]. In scalar field theories, this phenomenon simply does not occur for regular boundaries. For the same reasons topological transitions do not occur for finite scale transformations since the flip of eigenvalues from -1 to +1 requires a change in the parameter $t$ of the flow from $-\infty$ to $\infty$ as in (24)).

\section{Conformal Invariance and boundary conditions}

In $1+1$ dimensions the theory of massless scalar fields is formally conformal invariant. However, boundary conditions might break this symmetry $[9,10,11]$.

Conformal invariance is only preserved if the boundary conditions are stable under the boundary renormalization group flow. The fixed points can easily be identified. For a complex scalar field, besides the above mentioned fixed points, which correspond to Dirichlet, Neumann and pseudo-periodic boundary conditions and obviously are conformal invariant, there are fixed points corresponding to quasi-periodic boundary conditions (17). They also preserve the conformal symmetry.

In $1+1$ dimensions this exhausts the whole set of conformal invariant boundary conditions. Any other boundary condition flows toward one of these fixed points. The most stable fixed point corresponds to Neumann conditions because all its critical exponents are +1 . The most unstable is that of Dirichlet conditions since all critical exponents -1 . This is compatible with the fact that the neighbourhood of Dirichlet boundary conditions is plagued of singularities

Periodic, quasi-periodic and pseudo-periodic fixed points present relevant and irrelevant perturbations with critical exponents \pm 1 , respectively. Negative values label the possible instabilities. Implications of these results for string theory are well known. Periodic boundary conditions, appear as attractors of systems with quasi-periodic and pseudo-periodic conditions which stresses the stability of closed string theory vacuum. For open strings the (stable) attractor points are standard free strings (Neumann). Any other boundary condition flow toward one of those fixed points.

Notice that the absence of topological transitions in the boundary renormalization group flow is a consequence of the fact that all relevant perturbations are always associated with -1 critical exponents. 
In higher dimensions $(D>1)$ conformal invariance requires, even in the massless case $m=0$, that Neumann boundary conditions have to be modified in order to preserve conformal invariance with a term

$$
\partial_{n} \varphi=\frac{D-1}{4 D} K \varphi,
$$

proportional to the extrinsic curvature $K$ of the boundary.

In the case of singular boundaries some more interesting boundary renormalization group flows arise (see e.g. [18] for a review): fixed points and cyclic orbits of the boundary renormalization group flow can appear [15, 25, 24] and conformal invariance can be partially broken to a discrete subgroup $\mathbb{Z}[24]$.

\section{Vacuum energy and boundary conditions}

The infrared properties of quantum field theory are very sensitive to boundary conditions [26]. In particular, physical properties of the quantum vacuum state like the vacuum energy may exhibit a very strong dependence on the type of boundary conditions. This can be explicitly shown in the simple case of a massless field defined on a finite one-dimensional interval $[0, L]$.

For pseudoperiodic boundary conditions defined by the unitary operator

$$
U_{\theta}=\cos \theta \quad \sigma_{x}-\sin \theta \quad \sigma_{y}: \quad \varphi(L)=\mathrm{e}^{i \theta} \varphi(0)
$$

the Casimir vacuum energy (see e.g. Ref. [5] and references therein) is given by

$$
E_{0}=\frac{\pi}{L}\left(\frac{1}{12}-\min _{n \in \mathbb{Z}}\left(\frac{\theta}{2 \pi}+n-\frac{1}{2}\right)^{2}\right)
$$

The vacuum energy dependence on $\theta$ is in this case relatively smooth. The only cuspidal point at $\theta=0$ corresponds to periodic boundary conditions. A completely regular behaviour is obtained for Robin boundary conditions

$$
U=\mathrm{e}^{2 \alpha i} \mathbb{I}: \quad \partial_{n} \varphi(0)=\tan \alpha \varphi(0), \partial_{n} \varphi(L)=\tan \alpha \varphi(L)
$$

which smoothly interpolate between Dirichlet $\left(\alpha=\frac{\pi}{2}\right)$ and Neumann $(\alpha=\pi)$ conditions when $\alpha$ is restricted to the interval $\alpha \in\left[\frac{\pi}{2}, \pi\right][27,28,29]$.

Finally, the Casimir energy for quasi-periodic boundary conditions [30]

$$
E_{0}=\frac{\pi}{L}\left(\frac{1}{12}-\min _{n \in \mathbb{Z}}\left(\frac{\alpha}{2 \pi}+n+\frac{1}{4}\right)^{2}\right)
$$

is also dependent on the choice of the parameter $\alpha$. Two particularly interesting cases are given by $\alpha=0, U_{Z}=\sigma_{z} ; \varphi(L)=0, \partial_{n} \varphi(0)=0$ and $\alpha=\pi$, $U_{Z}^{\prime}=\sigma_{z} ; \quad \varphi(0)=0, \partial_{n} \varphi(L)=0$ which correspond to a Zaremba (mixed) boundary conditions: one boundary is Dirichlet and the other Neumann. 


\section{Vacuum entanglement entropy}

The dependence of vacuum energy on boundary conditions seems to suggest that many other observables may suffer the same effect. In particular, one may wonder whether or not the entropy of the system is dependent on the type of conditions that constrain the values of the fields at the boundary. The entropy of the field theory at finite temperature scales with the volume of physical space. Only in quantum gravity or string theory the entropy can scale with the area of black hole horizon. However, in field theory it is possible

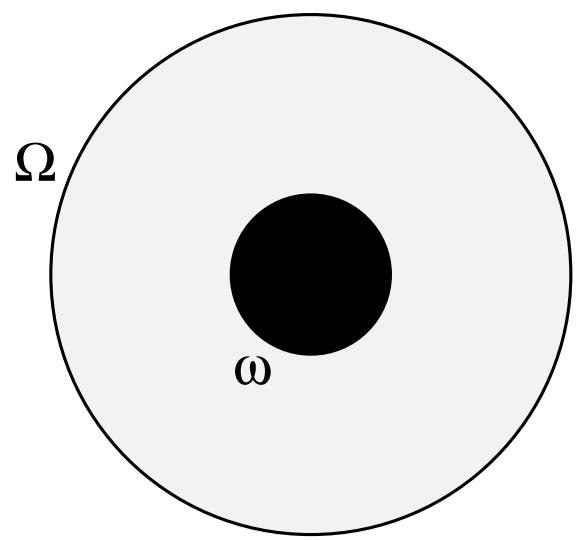

Figure 1. Information loss by integration over the fluctuations of the fields inside the domain $\omega$ to generate a mixed state from the pure vacuum state $\Psi_{0}$ by integrating out the fluctuating modes in a bounded domain $\omega$ of the physical space $\Omega$ (see Figure 1)

$$
\rho_{\omega}=\int_{\omega} \Psi_{0}^{*} \Psi_{0}
$$

The entropy of this state $S_{\omega}=-\operatorname{Tr} \rho_{\omega} \log \rho_{\omega}$, although ultravioletly divergent, provides a measure of the degree of entanglement of the vacuum state. In the case of a free massless real scalar field theory in one-dimensional spaces $(D=1)$ this entropy scales logarithmically with the size $l_{\omega}$ of $\omega$ and the ultraviolet cut-off $\epsilon$ introduced to split apart the domain $\omega$ and its complement $\Omega \backslash \omega$

$$
S_{\omega}=\frac{1}{3} \log \frac{l_{\omega}}{\epsilon}
$$

and in $D=2$ dimensions it scales linearly with the perimeter $R_{\omega}$ of $\omega$

$$
S_{\omega}=c_{2} \frac{R_{\omega}}{\epsilon}-\gamma
$$

and in $D>2$ dimensions as the volume of the boundary of $\omega$

$$
S_{\omega}=c_{D} V_{\omega} \epsilon^{1-D} .
$$

In particular in three-dimensional spaces it scales with the area of the boundary of $\omega$ like in the presence of a blackhole $[16,17]$. Although the coefficients of the leading terms $c_{2}, c_{D}$ in (32) and (33) have been explicitly computed, they are not universal because they obviously depend on the choice of the UV cutoff $\epsilon$. On the contrary, the coefficient 
$c_{1}=1 / 3$ of the logarithmic term in (31) is universal and does coincide with one third of the central charge of the corresponding conformal field theory. Similarly, the finite $\gamma$ term in (32) is also universal in $D=2$ dimension and is related to a degree of topological entanglement [31].

It is remarkable that in $D=1$ the coefficient $c_{1}=1 / 3$ is also independent of the choice of boundary condition in $\Omega$. This in contrast with what happens for the finite size corrections to vacuum energy. The coefficient of the $1 / L$ term is also proportional to the central charge but in that case the corresponding factor is very sensitive to the type of boundary conditions imposed at the boundary of $\Omega$. The above results indicate that whereas the Casimir energy is closely related with the infrared properties of the conformal theory which are sensitive to the boundary conditions, the entanglement entropy is rather associated to the behaviour at the interface between $\omega$ and its complement $\Omega \backslash \omega$ which do not depend on the choice of boundary conditions at the edge of the physical space.

\section{Conclusions}

The description of regular boundary conditions in terms of unitary matrices provides a very useful framework for the description of the boundary renormalization group flow and the breaking of conformal invariance due to boundary effects. Neumann conditions turn out to be the only boundary conditions which are absolutely stable under RG flow. All other boundary conditions may have some relevant perturbations which are the source of RG instabilities. However, the global structure of the flow does not permit topological transitions.

The finite size corrections to vacuum energy are very sensitive to the choice of boundary conditions which discriminate between the different fixed points of the renormalization group flow. On the contrary, the leading contribution to entanglement entropy of the vacuum is insensitive, for one-dimensional massless scalar field theories, to the change of boundary conditions. In $\mathrm{D}=2$ dimensions the same property holds for the finite correction to the entanglement entropy of massless scalar theories. This fact, is very relevant for the implementation of quantum codes with topological stability [31]. However, these properties do not hold for the leading terms contributing to the entanglement entropy.

\section{Acknowledgements}

We thank E. Elizalde, J.G. Esteve, S. Odintsov and G. Sierra for interesting discussions on closely related subjects. This work is partially supported by CICYT (grant FPA2004-02948) and DGIID-DGA (grant2006-E24/2). 


\section{References}

[1] T. Padmanabhan, Phys. Rept. 380 (2003) 235

[2] D. Deutsch, P. Candelas, Phys. Rev. D20 (1979) 3063

[3] H. B. G. Casimir, Proc. K. ned. Akad. Wet. 51(1948) 793

[4] K. A. Milton, The Casimir Effect: Physical Manifestations of the Zero Point Energy, World Sci., Singapore (2001)

[5] M. Bordag, U. Mohideen, V. M. Mostepanenko, Phys. Rep. 353 (2002) 1

[6] M. Asorey, D. García-Alvarez and J.M. Muñoz-Castañeda, J. Phys. A 39 (2006) 6127

[7] I. G. Moss, Class. Quant. Grav. 6 (1989) 759

[8] S. D. Odintsov, Class. Quant. Grav., 7(1990)445

[9] I. Affleck, Nucl. Phys. (Proc. Suppl.) B58 (1997) 35

[10] J. B. Zuber and V. B. Petkova, Arxive preprint [hep-th/0103007]

[11] J. Cardy, In Encyclopedia of Mathematical Physics, Eds. J.-P. Françoise, G. L. Naber and T. S. Tsun, Academic Press (2006)

[12] J. Polchinski, Phys. Rev. Lett. 75 (1995) 4724

[13] M. Asorey, A. Ibort and G. Marmo, Int. J. Mod. Phys. A 20 (2005) 1001

[14] A. P. Balachandran, G. Bimonte, G. Marmo and A. Simoni, Nucl. Phys. B 446 (1995) 299

[15] S. D. Glazek and K. G. Wilson, Phys. Rev. Lett. 89 (2002) 230401

[16] L. Bombelli, R.K. Koul, J. Lee and R. Sorkin, Phys. Rev. D 34 (1986) 373

[17] M. Srednicki, Phys. Rev. Lett. 71 (1993) 666

[18] I. Tsutsui and T. Fülöp, Arxive preprint [quant-ph/0312028]

[19] M. Bordag, H. Falomir, E. M. Santangelo and D. V. Vassilevich, Phys.Rev. D65 (2002) 064032

[20] A. A. Saharian, Phys. Rev. D69 (2004) 085005

[21] K. Symanzik, Nucl. Phys. B190 (1981) 1

[22] E. Elizalde, J. Phys. A 36 (2003) L567

[23] N.Graham, R.L.Jaffe, V.Khemani, M.Quandt, O. Schroeder and H.Weigel, Nucl.Phys. B677 (2004) 379-404

[24] M. Asorey, J.G. Esteve and G. Sierra, In preparation (2006)

[25] A. LeClair, J. M. Roman and G. Sierra, Phys. Rev. B69 (2004) 20505

[26] M.Asorey, J. Geom. Phys. 11(1993)94

[27] A. Romeo and A. A. Saharian, J. Phys. A35 (2002) 1297

[28] L. C. Albuquerque, R. M. Cavalcanti, J.Phys. A37 (2004) 7039

[29] B. Mintz, C. Farina, P. A. Maia Neto and R. B. Rodrigues, J. Phys. A39 (2006) 11325

[30] M. Asorey, unpublished (1994)

[31] A. Kitaev and J. Preskill, Phys. Rev. Lett. 96 (2006) 110404 